\title{
On the Behavior of the Generalized Alignment Index (GALI) Method for Regular Motion in Multidimensional Hamiltonian Systems
}

\author{
H. T. Moges* \\ Department of Mathematics and Applied Mathematics, \\ University of Cape Town, Rondebosch, 7701, Cape Town, SOUTH AFRICA \\ Th. Manos ${ }^{\dagger}$ \\ Laboratoire de Physique Théorique et Modélisation, CY Cergy Paris Université, \\ CNRS, UMR 8089, 95302 Cergy-Pontoise cedex, FRANCE \\ Ch. Skokos ${ }^{\ddagger}$ \\ Department of Mathematics and Applied Mathematics, \\ University of Cape Town, Rondebosch, 7701 Cape Town, SOUTH AFRICA
}

(Received 28 November, 2019)

\begin{abstract}
We investigate the behavior of the Generalized Alignment Index of order $k\left(\mathrm{GALI}_{k}\right)$ for regular orbits of multidimensional Hamiltonian systems. The $\mathrm{GALI}_{k}$ is an efficient chaos indicator, which asymptotically attains positive values for regular motion when $2 \leq k \leq N$, with $N$ being the dimension (D) of the torus on which the motion occurs. By considering several regular orbits in the neighborhood of two typical simple, stable periodic orbits of the Fermi-Pasta-Ulam-Tsingou (FPUT) $\beta$ model for various values of the system's degrees of freedom, we show that the asymptotic $\mathrm{GALI}_{k}$ values decrease when the order $k$ of the index increases and when the orbit's energy approaches the periodic orbit's destabilization energy where the stability island vanishes, while they increase when the considered regular orbit moves further away from the periodic one for a fixed energy. In addition, by performing extensive numerical simulations we show that the behavior of the index does not depend on the choice of the initial deviation vectors needed for its evaluation.
\end{abstract}

PACS numbers: 05.45.-a, 05.45.Ac, 05.90. $+\mathrm{m}$

Keywords: dynamical system, hamiltonian, generalized alignment index, periodic orbit

DOI: https://doi.org/10.33581/1561-4085-2020-23-2-153-164

\section{Introduction}

Dynamical systems constitute a rather substantial research area with many different applications in several fields of physics. In particular, Hamiltonian systems with many degrees of freedom (dof) have been broadly used for the study and better understanding of energy transport and equipartition phenomena (see for example [1-5]). The latter phenomena are associated with the dynamical nature of the motion fostered in the respective phase space, namely the regular or chaotic evolution of the system's orbits [3].

The rapid and efficient detection of the

\footnotetext{
${ }^{*}$ E-mail: mgshen002@myuct.ac.za

${ }^{\dagger}$ E-mail: thanos.manos@cyu.fr

${ }^{\ddagger}$ E-mail: haris.skokos@uct.ac.za
}

regular or chaotic nature of motion in many dof systems has been a very active research topic over the years, starting with the theoretical introduction of Lyapunov Exponents (LEs) [6, 7], and the development of efficient algorithms for their calculation [8,9] (see also [10] for a recent review), which in time expanded and evolved as a subfield of dynamical systems leading to a gallery of chaos detection methods (see e.g. [11-13] for comparisons among different methods and [14] for a collection of recent reviews of several chaos indicators).

In this study, we employ the Generalized Alignment Index (GALI) [15], a chaos detection method which has been shown to be a very efficient and fast tool for such purposes, especially in high dimensional problems (see e.g. [16, 17]). GALI's main advantages are (i) the quick distinction between regular and chaotic motion, (ii) the early detection of weak chaotic behavior, 
(iii) the determination of the dimensionality of the torus on which the quasi-periodic motion occurs, and (iv) the prediction of slow diffusion [16].

The GALI requires the evolution of several deviation vectors from a given orbit. The number $k$ of the vectors used defines the order of the index i.e. $\mathrm{GALI}_{k}$. In the case of regular motion $\mathrm{GALI}_{k}$, for $k$ not greater than the dimension $N$ of the tangent space of the torus on which the motion takes place, eventually oscillates around a positive value, while for chaotic orbits it tends exponentially fast to zero [15]. In the case of stable periodic orbits of Hamiltonian systems, GALI $_{k}$ tends to zero following particular power laws, while for unstable periodic orbits it becomes zero exponentially fast [18]. A detailed discussion of the theory of GALIs, along with their applications to various dynamical systems can be found in [19].

In this paper, we seek out to investigate and understand the GALIs' prediction efficiency and performance when the initial condition (IC) of an orbit moves gradually from a regular motion region to a chaotic one in Hamiltonian systems, by studying in detail the variation of the asymptotic values of the indices. This transition can take place either by changing the total energy of the system or by moving the regular orbit's IC towards the edge of a stability island. In addition, we follow the time evolution of the deviation vectors needed for the computation of the GALIs and examine if different initial distributions of the coordinates of these vectors affects the final GALI value. We perform our investigations in phase space regions around some simple, stable periodic orbits of the Fermi-Pasta-Ulam-Tsingou (FPUT) model [20, 21], which describes a chain of harmonic oscillators coupled through nonlinear interactions. The dynamics of this system has been studied extensively in the last decades (see e.g. [22, 23] and references therein) and is considered nowadays a prototypical, multidimensional, nonlinear model.

The paper is organized as follows. In Section 2 we present the FPUT $\beta$ system, discuss the numerical integration of its equations of motion and its variational equations, and briefly present the definition of the GALI method, its properties, as well as its computation. Then, in Section 3 we study in detail the asymptotic behavior of the GALIs for regular orbits in the phase space neighborhood of two types of stable periodic orbits of the FPUT $\beta$ model considering systems with different dof. In Section 4 we focus our attention on the properties of the deviation vectors used for the computation of the GALIs, while in Section 5 we summarize our results and discuss our findings.

\section{Model and numerical techniques}

In our study, we consider the onedimensional (1D) FPUT $\beta$ model [20, 21], which represents a lattice chain of $N$ identical particles with quartic nearest-neighbor interactions. The system's Hamiltonian function is given by

$$
\begin{aligned}
H_{N} & =\sum_{i=1}^{N} \frac{1}{2} p_{i}^{2} \\
& +\sum_{i=0}^{N}\left[\frac{1}{2}\left(x_{i+1}-x_{i}\right)^{2}+\frac{\beta}{4}\left(x_{i+1}-x_{i}\right)^{4}\right],
\end{aligned}
$$

where $x_{i}$ is the displacement of the $i$ th particle from its equilibrium position and $p_{i}$ is the corresponding conjugate momentum. In our investigation we impose fixed boundary conditions to system (1), i.e. $x_{0}=x_{N+1}=p_{0}=p_{N+1}=0$ and set $\beta=1$. The time evolution of a phase space orbit with ICs at time $t=t_{0} \vec{z}\left(t_{0}\right)=\left(\vec{x}\left(t_{0}\right), \vec{p}\left(t_{0}\right)\right)$, where $\vec{x}\left(t_{0}\right)=\left(x_{1}\left(t_{0}\right), x_{2}\left(t_{0}\right), \ldots, x_{N}\left(t_{0}\right)\right)$ and $\vec{p}\left(t_{0}\right)=\left(p_{1}\left(t_{0}\right), p_{2}\left(t_{0}\right), \ldots, p_{N}\left(t_{0}\right)\right)$, is governed by the Hamilton equations of motion $\dot{x_{i}}=\frac{d x_{i}}{d t}=\frac{\partial H_{N}}{\partial p_{i}}, \dot{p}_{i}=\frac{d p_{i}}{d t}=-\frac{\partial H_{N}}{\partial x_{i}}$, $i=1,2, \ldots, N$.

In order to compute the $\mathrm{GALI}_{k}$ chaos indicator for a particular orbit we have to follow the evolution of the orbit itself, along with a set of $k$, initially linearly independent, deviation vectors $\vec{v}_{i}, i=1,2, \ldots, k$, corresponding to $k$ different perturbations of the studied orbit. The time evolution of an initial deviation vector $\vec{v}\left(t_{0}\right)=\delta \vec{z}\left(t_{0}\right)=\left(\delta \vec{x}\left(t_{0}\right), \delta \vec{p}\left(t_{0}\right)\right)=$ $\left(\delta x_{1}\left(t_{0}\right), \ldots, \delta x_{N}\left(t_{0}\right), \delta p_{1}\left(t_{0}\right), \ldots, \delta p_{N}\left(t_{0}\right)\right)$ from an orbit with ICs $\vec{z}\left(t_{0}\right)=\left(\vec{x}\left(t_{0}\right), \vec{p}\left(t_{0}\right)\right)$ is governed by the so-called variational equations (see e.g. [10])

$$
\dot{\vec{v}}(t)=\left[\mathbf{J}_{2 N} \cdot \mathbf{D}_{H}^{2}(z(t))\right] \cdot \vec{v}\left(t_{0}\right),
$$

where $\mathbf{J}_{2 N}=\left[\begin{array}{cc}\mathbf{0}_{N} & \mathbf{I}_{N} \\ -\mathbf{I}_{N} & \mathbf{0}_{N}\end{array}\right]$, with $\mathbf{I}_{N}$ and $\mathbf{0}_{N}$ being respectively the identity and the zero $N \times N$ 
matrices, and $\mathbf{D}_{H}^{2}(z(t))$ is the $2 N \times 2 N$ Hessian matrix with elements $\mathbf{D}_{H}^{2}(z(t))_{i, j}=\left.\frac{\partial^{2} H}{\partial z_{i} \partial z_{j}}\right|_{z(t)}$, $i, j=1,2, \ldots, 2 N$.

Since Hamiltonian (1) can be split in two integrable parts $H_{N}(\vec{x}, \vec{p})=A(\vec{p})+B(\vec{x})$, with $A(\vec{p})$ being the kinetic energy, which is a function of only the momenta $p_{i}$, and $B(\vec{x})$ being the potential energy depending only on the coordinates $x_{i}$, we implement an efficient fourth-order symplectic integration scheme called ABA864 [24-26] for integrating the system's equations of motion. Symplectic integrators are numerical schemes specifically designed to preserve the symplectic structure of Hamiltonian systems. One of their main advantages is that they keep the error in the computed value of the Hamiltonian (which is typically referred to as the system's 'energy') bounded for all times. In our numerical simulations, we typically integrate system (1) up to a final time $t_{f}=10^{8}$. By adequately adjusting the used integration time step we always keep the absolute value of the relative energy error below $10^{-8}$. By implementing the so-called 'tangent map' technique [27-29] we also use the ABA864 scheme for integrating the system's variational equations (2), following in this way the time evolution of the set of deviation vectors needed for the computation of the GALIs.

\subsection{The GALI method}

Let us now briefly discuss the GALI method and its properties considering our $N$ dof autonomous Hamiltonian (1). By following an orbit $\vec{z}(t)=(\vec{x}(t), \vec{p}(t))$ and a set of $k$ initially independent deviation vectors $\vec{v}_{i}(t)$, $i=1,2, \ldots, k$, with $2 \leq k \leq 2 N$, the Generalized Alignment Index of order $k\left(\mathrm{GALI}_{k}\right)$ represents at any time $t$ the volume of the generalized parallelogram having as edges the $k$ unit deviation vectors $\hat{v}_{i}(t)=\frac{\vec{v}_{i}(t)}{\left\|\vec{v}_{i}(t)\right\|}[15]$. This volume is computed as the norm of the wedge product of these vectors

$$
\operatorname{GALI}_{k}(t)=\left\|\hat{v}_{1}(t) \wedge \hat{v}_{2}(t) \wedge \ldots \wedge \hat{v}_{k}(t)\right\| .
$$

We note that the number $k$ of the considered deviation vectors should not exceed the dimension $2 N$ of the system's phase space, because in that case the vectors will be by default linearly dependent, and the corresponding volume (and consequently the value of $\mathrm{GALI}_{k}$ ) will be zero. The GALI is a generalization of the Smaller Alignment Index (SALI) [30-32], with GALI $_{2}$ being equivalent to SALI [15]. Both indices have been proven to be very efficient chaos indicators and have been successfully implemented in studies of various dynamical systems (see e.g. [19] and references therein).

In an $N$ dof Hamiltonian system regular motion typically occurs on ND tori. The system's dynamics leads any deviation vector of the regular orbit to eventually fall on the ND tangent space of the torus [15]. Thus, asymptotically the volume defined by $k$ initially linearly independent deviation vectors, with $N<k \leq 2 N$, will vanish as these vectors will become linearly dependent, while, in general, for $2 \leq k \leq N$ the volume will not become zero as the vectors will remain linearly independent. The general behavior of the $\mathrm{GALI}_{k}$ for regular orbits lying on an $N D$ torus of an $N$ dof Hamiltonian system is given by [15]

$$
\operatorname{GALI}_{k}(t) \propto\left\{\begin{array}{l}
\text { constant } \text { if } 2 \leq k \leq N \\
t^{-2(k-N)} \text { if } N<k \leq 2 N .
\end{array}\right.
$$

We note that the power law decay in (4) changes in the case of motion on a low-dimensional torus [16] (i.e. a torus with dimensionality lower than $N)$, whose existence in the FPUT $\beta$ model was shown in [33], and if some of the initial deviation vectors are already on the torus' tangent space [15], but we will not discuss such cases here. Tori of regular motion exist around stable periodic orbits in conservative (or Hamiltonian) systems. For such orbits, $\mathrm{GALI}_{k}$ always decreases to zero following some specific power laws [18]

$$
\operatorname{GALI}_{k}(t) \propto \begin{cases}t^{-(k-1)} & \text { if } 2 \leq k \leq 2 N-1 \\ t^{-2 N} & \text { if } k=2 N\end{cases}
$$

On the other hand, for chaotic orbits and unstable periodic orbits $\mathrm{GALI}_{k}$ decays exponentially fast to zero $[15,18]$

$$
\operatorname{GALI}_{k} \propto e^{-\left[\left(\sigma_{1}-\sigma_{2}\right)+\left(\sigma_{1}-\sigma_{3}\right)+\cdots+\left(\sigma_{1}-\sigma_{k}\right)\right] t},
$$

where $\sigma_{1} \geq \sigma_{2} \geq \cdots \geq \sigma_{k}$ are approximations of the first $k$ largest LEs of the orbit.

An efficient way of computing the value of $\mathrm{GALI}_{k}$ is through the Singular Value 
Decomposition (SVD) of the matrix

$\mathbf{A}=\left(\begin{array}{llll}\hat{v}_{1} & \hat{v}_{2} & \cdots & \hat{v}_{k}\end{array}\right)=\left(\begin{array}{cccc}\hat{v}_{1,1} & \hat{v}_{2,1} & \cdots & \hat{v}_{k, 1} \\ \hat{v}_{1,2} & \hat{v}_{2,2} & \cdots & \hat{v}_{k, 2} \\ \vdots & \vdots & & \vdots \\ \hat{v}_{1,2 N} & \hat{v}_{2,2 N} & \cdots & \hat{v}_{k, 2 N}\end{array}\right)$

having as columns the coordinates of the $k$ unitary vectors $\hat{v}_{i}(t)=\frac{\vec{v}_{i}(t)}{\left\|\vec{v}_{i}(t)\right\|}=$ $\left(\hat{v}_{i, 1}, \hat{v}_{i, 2}, \ldots, \hat{v}_{i, 2 N}\right)[16]$. In particular,

$$
\operatorname{GALI}_{k}=\prod_{i=1}^{k} z_{i}
$$

where $z_{i}, i=1, \ldots, k$, are the so-called singular values of $\mathbf{A}$, obtained through the SVD procedure.

We compute the value of $\mathrm{GALI}_{k}$ by following the time evolution of $k$ initially independent random unit deviation vectors $\hat{v}_{1}(0), \hat{v}_{2}(0), \ldots, \hat{v}_{k}(0)$. In order to statistically analyze the behavior of GALIs we average the indices over several different choices of the set of initial deviation vectors. The random choice of the initial vectors leads to different $\mathrm{GALI}_{k}(0)$ values. Thus, in order to fairly and adequately compare the behavior of the indices for different initial sets of vectors we normalize the GALIs evolution by registering the ratio $\operatorname{GALI}_{k}(t) / \mathrm{GALI}_{k}(0)$, i.e. we measure the change of the volume defined by the $k$ deviation vectors with respect to the initially defined volume. Another option is to start the evolution of the dynamics by considering a set of $k$ orthonormal vectors so that $\operatorname{GALI}_{k}(0)=1$. As we will see later on, both approaches lead to similar results, so in our study we will follow the former procedure, unless otherwise stated. In order to illustrate the basic behavior of the GALIs for regular and chaotic orbits we present in Figure 1(a) [Figure 1(b)] the time evolution of GALIs for a regular [chaotic] orbit of Hamiltonian (1) with $N=5$. The regular orbit of Figure 1(a) lies on a $5 \mathrm{D}$ torus and consequently $\mathrm{GALI}_{2}$, $\mathrm{GALI}_{3}, \mathrm{GALI}_{4}$ and $\mathrm{GALI}_{5}$ eventually oscillate around some constant non-zero value, which decreases with increasing $k$. One of our main aims in this work is to analyze in depth this behavior. Note that the saturation of the indices to their practically constant limiting values happens at latter times for higher $k$ values. The GALIs with $k \geq 6$ eventually tend to zero following asymptotic power laws, which are indicated by straight lines in Figure 1(a). All these behaviors are in accordance with Equation (4). On the other hand, as expected from (6) the GALIs decay exponentially fast to zero in the case of the chaotic orbit of Figure 1(b). Since we focus here on the behavior of GALIs for regular orbits, we do not investigate further the case of Figure 1(b), by e.g. computing the corresponding LEs. Such investigations were systematically performed, for example, in $[15,16]$.

\section{The behavior of the GALI for regular orbits}

In general, regular motion occurs in the vicinity of stable periodic orbits in Hamiltonian systems. Thus, in order to study the behavior of the GALIs for regular orbits we first locate some stable periodic orbits of system (1) and then compute the GALIs for orbits in the neighborhood of these orbits. The stability type of a periodic orbit is determined by the eigenvalues of the so-called monodromy matrix $\mathbf{M}(T)$, which is obtained from the solution of the variational equations of the periodic orbit for one period $T$ (for more details, explicit equations and explanations see e.g. [34] and references therein). The monodromy matrix is symplectic, and its columns are linearly independent solutions of the equations that govern the evolution of deviation vectors from the periodic orbit. If all the eigenvalues of $\mathbf{M}(T)$ are on the unit circle in the complex plane the corresponding periodic orbit is stable, while otherwise, it is unstable. We note that there exist several different types of instabilities [34], but we will not discuss this issue here.

In an ND autonomous Hamiltonian system, two eigenvalues are always equal to $\lambda=1$ [34], which means that in practice the remaining $2(N-1)$ eigenvalues define the periodic orbit's stability. Thus, we can reduce our investigation to a $2(N-1)$ D subspace of the whole phase space through the well-known method of the Poincaré Surface of Section (PSS) (see e.g. [3]), where the corresponding monodromy matrix has $2(N-1)$ eigenvalues, none of which is by default $\lambda=1$.

In what follows we investigate the behavior of the GALIs for regular orbits in the neighborhood of two simple periodic orbits (SPOs) of system (1), which we refer to as SPO1 


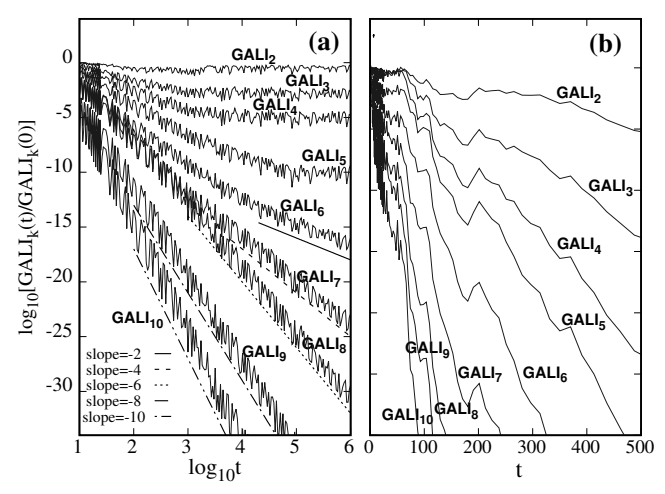

FIG. 1. The time evolution of the normalized GALI indices $\operatorname{GALI}_{k}(t) / \mathrm{GALI}_{k}(0), k=2,3, \ldots, 10$ for (a) a regular orbit with ICs $x_{1}=-1.03003$, $x_{3}=-x_{5}=1.04003, p_{1}=0.29284$, and (b) a chaotic orbit with ICs $x_{1}=1.00097, x_{3}=-x_{5}=1.04003$ $p_{1}=0.57060$, of system (1) with $N=5$ and total energy $H_{5}=5$. All the other coordinates of both orbits are initially set to zero. The straight lines in (a) correspond to functions proportional to $t^{-2}, t^{-4}, t^{-6}$, $t^{-8}$ and $t^{-10}$, as indicated in the panel's legend. Both axes of (a) and the vertical axis of (b) are logarithmic.

and SPO2. The dynamics of these orbits was discussed in $[35,36]$.

\subsection{Regular motion in the neighborhood} of SPO1

The first SPO we study is called SPO1 in [36] and it is obtained by considering an FPUT $\beta$ lattice (1) with $N$ being an odd integer, so that all particles at even-numbered positions are kept stationary at all times, while the odd-numbered particles are always displaced symmetrically to each other, i.e.

$$
\hat{x}_{2 j}(t)=0, \quad \hat{x}_{2 j-1}(t)=-\hat{x}_{2 j+1}(t)=\hat{x}(t),
$$

for $j=1,2, \ldots, \frac{N-1}{2}$. By inserting conditions (9) in the system's equations of motion we end up with a second order nonlinear differential equation for variable $\hat{x}(t)$ which describes the oscillations of all moving particles of the SPO1. In particular, for $j=1,3,5, \ldots, N$ we have

$$
\ddot{\hat{x}}_{j}(t)=-2 \hat{x}(t)-2 \beta \hat{x}^{3}(t),
$$

while $\hat{x}_{j}(t)=0, j=2,4,6, \ldots,(N-1)$ for all times.

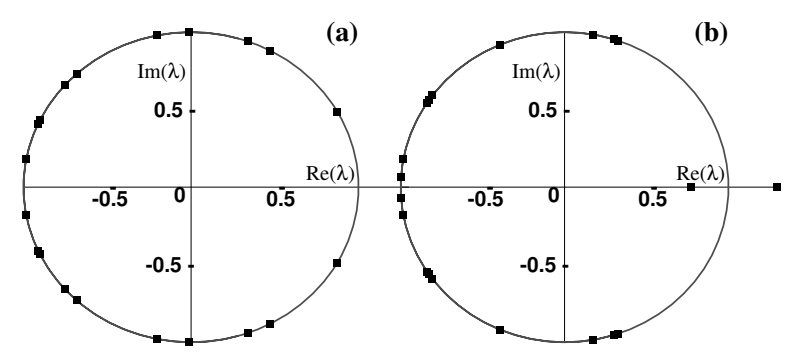

FIG. 2. The arrangement on the complex plane of the eigenvalues $\lambda_{i}, i=1,2, \ldots, 20$, of the monodromy matrix of the SPO1 (9) of Hamiltonian (1) with $N=11$ for (a) the stable SPO1 with $H_{N} / N=0.1$ and $\hat{x}(0)=-0.4112$, and (b) the unstable SPO1 with $H_{N} / N=0.2$ and $\hat{x}(0)=-0.5626$. The monodromy matrix is evaluated on the PSS (a) $x_{1}=-0.411$, $p_{1}>0$, and (b) $x_{1}=-0.560, p_{1}>0$. The critical energy density for which the SPO1 encounters its first transition from stability to instability is $H_{N}^{c} / N \approx 0.1755$.

The stability analysis of the SPO1 [35, 36] showed that for small values of $H_{N}$ (1) the orbit is stable, but it becomes unstable when the energy increases beyond a certain threshold $H_{N}^{c}$. More specifically, in [35] it was shown that the energy density threshold $H_{N}^{c} / N$ decreases with increasing $N$ following an asymptotic law $H_{N}^{c} / N \propto N^{-1}$ (see for example Figure 1 of [35]).

In Figure 2 we see the arrangement of the eigenvalues of the monodromy matrix of the SPO1 orbit of Hamiltonian (1) with $N=11$ when $H_{N} / N=0.1$ [Figure 2(a)] and $H_{N} / N=0.2$ [Figure 2(b)]. In the first case, the SPO1 is stable as all eigenvalues are on the unit circle, while in the latter the orbit is unstable as two eigenvalues are off the unit circle. We note that the transition from stability to instability for the SPO1 happens at $H_{N}^{c} / N \approx 0.1755$.

To investigate how GALIs of different orders $k$ behave for regular orbits we perturb the stable SPO1 with ICs $\hat{x}_{i}(0), \hat{p}_{i}(0), i=1,2, \ldots, N$ and energy density $H_{N} / N$ to obtain a nearby orbit with ICs $X_{i}(0), P_{i}(0)$, ensuring that the new orbit is at the same energy density level. The phase space distance $D$ between these two orbits, in this case, is given by

$D=\left\{\sum_{i=1}^{N}\left[\left(\hat{x}_{i}(0)-X_{i}(0)\right)^{2}+\left(\hat{p}_{i}(0)-P_{i}(0)\right)^{2}\right]\right\}^{1 / 2}$ 
Having this regular orbit lying on a $N D$ torus means that $\mathrm{GALI}_{k}$ with $2 \leq k \leq N$ will asymptomatically approach some almost constant value [see Equation (4)]. In order to statistically analyze these asymptotic $\mathrm{GALI}_{k}$ values we follow the evolution of GALIs for $n_{v}=10$ different random sets of initial unit deviation vectors whose coordinates are drawn from a uniform distribution in the interval $[-0.5,0.5]$, and compute the evolution of the average (over the $n_{v}$ sets of deviation vectors) values $\left\langle\operatorname{GALI}_{k}(t) / \operatorname{GALI}_{k}(0)\right\rangle$. The integration of the orbit and of the deviation vectors is performed until the GALI values show a clear saturation. Then we estimate the asymptotic GALI value by finding the mean value of $\left\langle\operatorname{GALI}_{k}(t) / \operatorname{GALI}_{k}(0)\right\rangle$ over the last $n_{t}=20$ recorded values, captured during, approximately, the one-fifth of the last decade of integration. We denote this quantity as $\overline{\mathrm{GALI}}_{k}$ and estimate its error through the standard deviation of the considered $n_{t}$ values.

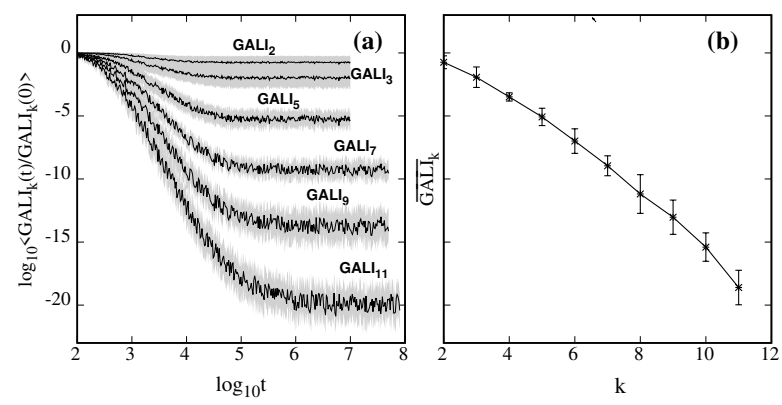

FIG. 3. (a) The time evolution of the average, over $n_{v}=10$ sets of random initial unit deviation vectors, $\left\langle\operatorname{GALI}_{k}(t) / \mathrm{GALI}_{k}(0)\right\rangle$ (black curves) for $k=2,3,5$, 7, 9 and 11, for a regular orbit in the vicinity of the stable SPO1 of system (1) with $N=11, H_{N} / N=0.01$ and $D=0.12$. The shaded gray area around each curve denotes 1 standard deviation. (b) Estimation of the asymptotic GALI values, $\overline{\mathrm{GALI}}_{k}$, as a function of their order $k$ for the orbit of panel (a). The error bars denote 1 standard deviation.

The outcome of this analysis for a regular orbit with distance (11) $D=0.12$ from the stable SPO1 of system (1) with $N=11$ and $H_{N} / N=0.01$ is seen in Figure 3. In particular, Figure 3(a) depicts the evolution of $\left\langle\operatorname{GALI}_{k}(t) / \mathrm{GALI}_{k}(0)\right\rangle$ for some selected orders $k$ (black curves). The gray area around these curves represents one standard deviation. We observe that GALIs of higher orders converge to lower values and need more time to settle to these values. In Figure 3(b) we see more clearly how these final asymptotic values, $\overline{\mathrm{GALI}}_{k}$, decrease with increasing $k$.

Let us now investigate how these behaviors change when the studied regular orbit is taken further and further away from the stable SPO1, i.e. as we increase $D$. A representative case is presented in Figure 4 where we plot the evolution of GALI $_{2}$ [Figure 4(a)] and GALI 4 [Figure 4(b)] for several orbits in the neighborhood of the stable SPO1 of Hamiltonian (1) with $N=11$, $H_{N} / N=0.01$ and $D_{1}=0.008, D_{2}=0.01$, $D_{3}=0.06, D_{4}=0.1, D_{5}=0.22$ and $D_{6}=0.4$, when one set of random initial deviation vectors is used. First of all, we observe that the behavior of the GALIs for the regular orbit closest to the SPO1 $\left(D_{1}=0.008\right)$ follows the same power laws as the periodic orbit itself, namely (5), i.e. $\mathrm{GALI}_{2} \propto t^{-1}$ and $\mathrm{GALI}_{4} \propto t^{-3}$. As $D$ increases, i.e. the IC of the regular orbit moves further away from the stable SPO1 inside the stability island surrounding the periodic orbit, GALIs start deviating from the power law decay observed for $D=D_{1}$, and finally saturate to a positive value. This deviation starts earlier for larger $D$ values and consequently the asymptotic values of GALIs increase as $D$ grows. Eventually, for very large values of $D$, in the particular case discussed in Figure 4 for $D=D_{6}=0.4$, the perturbed orbit becomes chaotic as it is located outside the stability island. In that case, the GALIs decrease exponentially fast to zero in accordance with (6).

In Figure 4 we show the increase of only the asymptotic values of $\mathrm{GALI}_{2}$ and $\mathrm{GALI}_{4}$ with respect to $D$. This behavior is clearly seen for the whole spectrum of GALIs in Figure 5 where we plot the $\overline{\mathrm{GALI}}_{k}$ values versus $k$ for regular orbits around the SPO1 orbit of system (1) with $N=11$ and $H_{N} / N=0.01$, having $D$ values which are relatively not too small, in order to avoid the power law decays observed in Figure 4 for $D=0.008$, but also relatively not too large in order for the studied orbit to be well inside the stability island.

So far we saw how the asymptotic GALI values change when we increase the distance $D$ of the studied orbit from the stable SPO1 for constant energy $H_{N}$ (and obviously constant energy density $\left.H_{N} / N\right)$. In order to see how $\overline{\mathrm{GALI}}_{k}$ values change when we increase the orbit's 

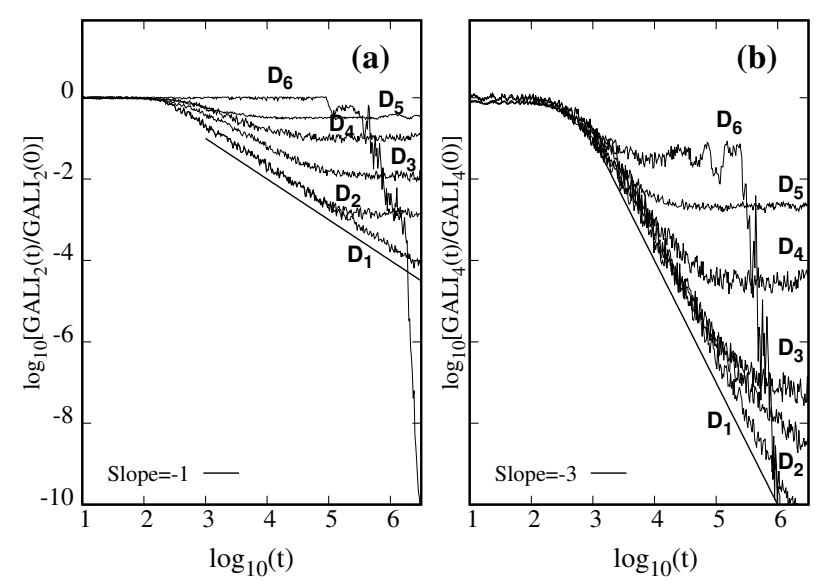

FIG. 4. The time evolution of (a) $\mathrm{GALI}_{2}(t) / \mathrm{GALI}_{2}(0)$ and (b) $\mathrm{GALI}_{4}(t) / \mathrm{GALI}_{4}(0)$ for orbits with $H_{N} / N=0.01$ and distances $D_{1}=0.008, D_{2}=0.01$, $D_{3}=0.06, D_{4}=0.1, D_{5}=0.22$ and $D_{6}=0.4$ from the stable SPO1 of Hamiltonian (1) with $N=11$, for 1 set of initial deviation vectors. The straight lines correspond to functions proportional to (a) $t^{-1}$, and (b) $t^{-3}$.

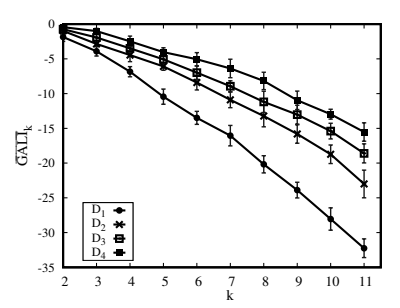

FIG. 5. Similar to Figure 3(b) but for regular orbits in the vicinity of the stable SPO1 of system (1) with $N=11, H_{N} / N=0.01$ and (a) $D_{1}=0.01, D_{2}=0.06$, $D_{3}=0.12$, and $D_{4}=0.22$.

energy density, we begin by estimating the size of the stability island around the SPO1 orbit with different $H_{N} / N$ values by finding the largest $D$ value for which regular motion is observed. We denote this value $D_{m}$. The outcome of this process is shown in Figure 6 . The $D_{m}$ value (and consequently the size of the stability island) decreases as $H_{N} / N$ grows, and vanishes when the SPO1 periodic orbit destabilizes and becomes unstable. Recall that in this case, the destabilization of the SPO1 takes place for $H_{N}^{c} / N \approx 0.1755$. Thus, the region below the curve of Figure 6 corresponds to regular motion.

In Figure 7 we present the values of $\overline{\mathrm{GALI}}_{k}$

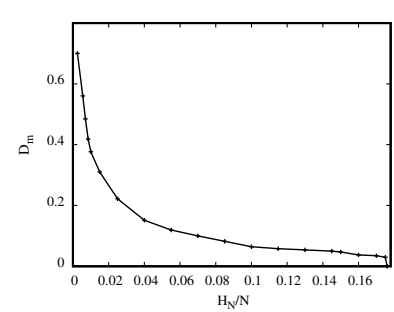

FIG. 6. Estimation of the extent in phase space of the stability island around SPO1: The maximum value of $D$, denoted by $D_{m}$, for which regular motion occurs, as a function of the energy density $H_{N} / N$ for the Hamiltonian (1) with $N=11$.

as a function of the GALI's order $k$ for regular orbits in the neighborhood of the stable SPO1 for increasing energy densities $E=H_{N} / N$, i.e. moving the orbit's IC in the region below the curve of Figure 6 towards the destabilization energy of SPO1. In particular, we consider regular orbits with $D=0.1$ for $E_{1}=0.01, E_{2}=0.04$ and with $D=0.01$ for $E_{3}=0.12$ and $E_{4}=0.16$. As the energy density increases the regular region in Figure 6 is shrinking, thus the considered regular orbit is located closer and closer to the stable SPO1 orbit itself and the $D$ value should also decrease in order to avoid transition to chaotic motion. Since the regular orbit approaches the stable SPO1 the $\overline{\mathrm{GALI}}_{k}$ values shown in Figure 7 decrease for orbits close to the periodic one, in accordance to what we observed in Figure 5.

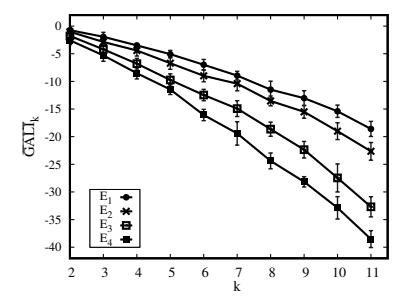

FIG. 7. Similar to Figure 5 but for regular orbits with different energy densities $E=H_{N} / N: E_{1}=0.01$, $E_{2}=0.04, E_{3}=0.12$ and $E_{4}=0.16$. The phase space distance $D(11)$ is $D=0.1$ for $E_{1}$ and $E_{2}$, and $D=0.01$ for $E_{3}$ and $E_{4}$.

The behavior we observe in the $N=11$ case for the asymptotic GALI values of regular orbits located further and further away from the stable SPO1 (Figure 5), as well as the one we see for regular orbits whose energies approach the destabilization energy of the SPO1 (Figure 7), 

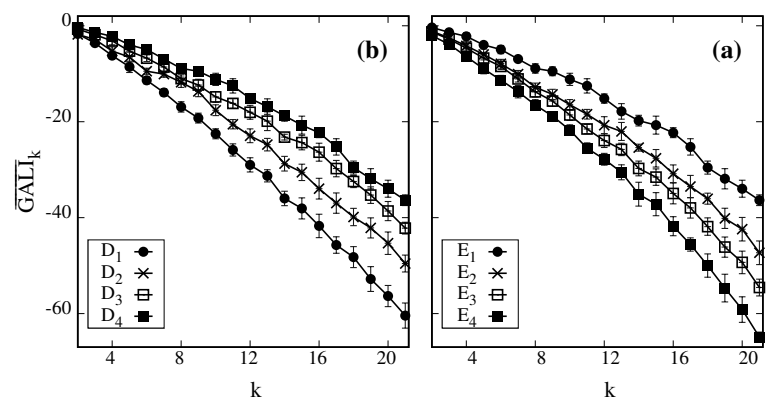

FIG. 8. Estimation of the asymptotic GALI values, $\overline{\mathrm{GALI}}_{k}$, as a function of their order $k$ for regular orbits in the vicinity of the stable SPO1 of system (1) with $N=21$. (a) Regular orbits for $E=H_{N} / N=0.002$ and distances $D$ (11) $D_{1}=0.01, D_{2}=0.04, D_{3}=0.08$ and $D_{4}=0.12$. (b) Regular orbits with $E_{1}=0.002$ and $D=0.12, E_{2}=0.006$ and $D=0.1, E_{3}=0.01$ and $D=0.09$, and $E_{4}=0.04$ and $D=0.03$. The error bars in both panels denote 1 standard deviation.

remain the same also for other values of $N$. As a testimony to that we present in Figure 8 results for the dependence of the $\overline{\mathrm{GALI}}_{k}$ values on the order $k$ of the index for the SPO1 orbit of system (1) with $N=21$. Note that in this case, the destabilization energy density of the SPO1 is $H_{N} / N \approx 0.0675$.

\subsection{Regular motion in the neighborhood} of $\mathrm{SPO} 2$

The findings of Section 3.1 are not restricted to only one type of SPO but are rather general. To demonstrate this we perform a similar analysis for regular orbits in the neighborhood of what was called SPO2 in [36]. This periodic orbit appears in FPUT $\beta$ systems (1) with $N=5+$ $3 m, m=0,1,2, \ldots$, particles where every third particle remains always stationary and the two particles in between move in opposite directions

$$
\begin{gathered}
\hat{x}_{3 j}(t)=0, \quad j=1,2,3, \ldots, \frac{N-2}{3}, \\
\hat{x}_{j}(t)=-\hat{x}_{j+1}(t)=\hat{x}(t), \\
j=1,4,7, \ldots, N-1 .
\end{gathered}
$$

Similarly to the case of SPO1 inserting conditions (12) and (13) in the equations of motion of system (1) leads to a single differential equation

$$
\ddot{\hat{x}}_{j}(t)=-3 \hat{x}(t)-9 \beta \hat{x}^{3}(t)
$$

for the moving particles, while $\hat{x}_{j}(t)=0$ for the stationary particles with $j=3,6,9, \ldots, N-2$. In [36], where the stability of this orbit was studied in detail, it was found that the destabilization energy density threshold $H_{N}^{c} / N$ decreases when the number of particles grows, as $H_{N}^{c} / N \propto N^{-2}$ (see Figure 2(a) of [36]).

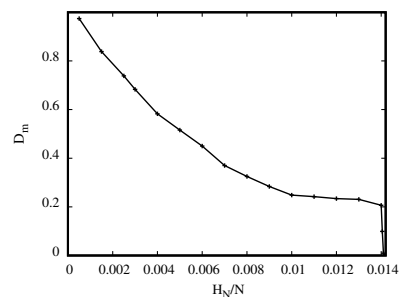

FIG. 9. Similar to Figure 6 but for the SPO2 of Hamiltonian (1) with $N=11$.
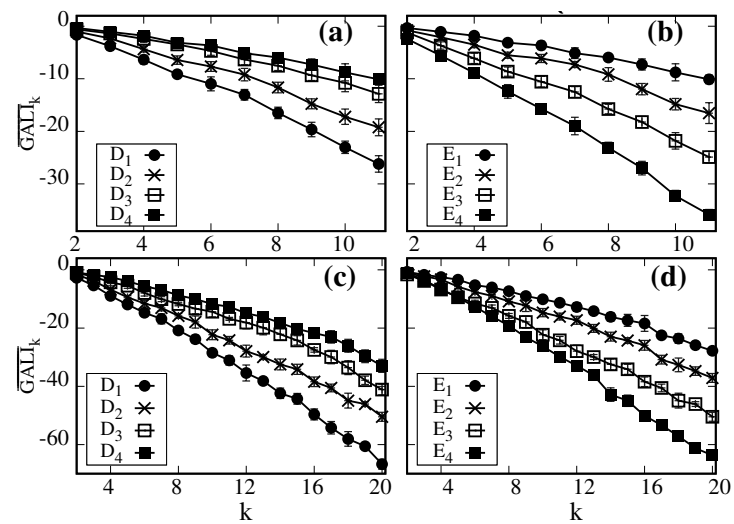

FIG. 10. Estimation of the asymptotic GALI values, $\overline{\mathrm{GALI}}_{k}$, as a function of their order $k$ for regular orbits in the vicinity of the stable SPO2 of system (1) with $N=11[(\mathrm{a})$ and (b)] and $N=20$ [(c) and (d)]. The considered regular orbits have (a) $E=H_{N} / N=0.001$ and distances $D$ (11) from the stable SPO2 $D_{1}=0.01, D_{2}=0.04, D_{3}=0.08$ and $D_{4}=0.12$, (c) $E=H_{N} / N=0.002$ with $D_{1}=0.01$, $D_{2}=0.04, D_{3}=0.08$ and $D_{4}=0.12$, (b) $E_{1}=0.001$ and $D=0.12, E_{2}=0.004$ and $D=0.12, E_{3}=0.008$ and $D=0.04$, and $E_{4}=0.012$ and $D=0.01$, (d) $E_{1}=0.0004$ and $D=0.1, E_{2}=0.0008$ and $D=0.04, E_{3}=0.002$ and $D=0.04$, and $E_{4}=0.004$ and $D=0.002$.

For $N=11$ the stability region of $\mathrm{SPO} 2$ is shown in Figure 9, which can be directly compared with the stability region of SPO1 in Figure 6, as both cases have the same number of 
particles. The shapes of both stability regions look similar but the extent of the SPO2 region in the energy density axis is much smaller as the stable SPO2 becomes unstable for $H_{N}^{c} / N \approx 0.01395$.

In Figure 10 we present results regarding the asymptotic GALI values, $\overline{\mathrm{GALI}}_{k}$, for regular orbits inside the stability island around the SPO2 orbit for $N=11$ [Figures 10(a) and (b)] and for $N=20$ [Figures $10(\mathrm{c})$ and (d)]. We observe similar behaviors to the ones encountered in the neighborhood of SPO1. In particular, the $\overline{\mathrm{GALI}}_{k}$ values increase as we move away from the stable periodic orbit [Figures 10(a) and (c)], similarly to what we observed in Figures 5 and 8(a), while they decrease as the energy of the regular orbit approaches the destabilization energy of SPO2 [Figures 10(b) and (d)] as we saw in Figures 7 and 8(b) for the SPO1 case. We note that for $N=20$ the destabilization threshold is $H_{N}^{c} / N \approx 0.00425$.

\section{Statistical analysis of deviation vectors}

In the case of chaotic orbits it is known that all, initially different, deviation vectors will eventually become aligned to the direction defined by the maximum LE [8-10]. This behavior leads to the eventual vanishing of the GALIs through the exponential decay (6) [15]. On the other hand, for regular orbits we know that deviation vectors eventually fall on the tangent space of the torus on which the motion occurs [15], but it is not clear how this behavior affects the actual GALI values. For this reason, we investigate here in detail the behavior of deviation vectors for regular orbits.

An important finding is that the evolution of the GALIs does not practically depend on the initial choice of deviation vectors needed for their computation. A particular example illustrating this property is shown in Figure 11 where we present the evolution of GALIs for a regular orbit close to the stable SPO1 $(D=0.12)$ of Hamiltonian (1) for $N=11$ and $H_{N} / N=0.01$. In Figure 11(a) we show the evolution of $\left\langle\operatorname{GALI}_{k}(t) / \operatorname{GALI}_{k}(0)\right\rangle$ for some selected values of $k$ when the coordinates of the unit initial deviation vectors are chosen from a uniform distribution in the interval $[-0.5,0.5]$ (black curves) or from a normal distribution with mean 0 and standard deviation 1 (light black curves). The two curves practically coincide. The presented results are averaged over $n_{v}=10$ random sets of initial deviation vectors and the gray area around the curves indicate one standard deviation, as was done for example in Figure 3(a). In Figure 11(b) we show a similar computation, but now the initial deviation vectors, whose initial coordinates were created in a similar way as in Figure 11(a), were orthonormalized at the beginning of their evolution. This choice sets $\mathrm{GALI}_{k}(0)=1$ and for this reason we report in Figure 11(b) the average value $\left\langle\operatorname{GALI}_{k}(t)\right\rangle$. Also for this case, we see that the method of creation of the initial, orthonormal deviation vectors does not affect the evolution of the GALIs. Furthermore, by comparing Figures 11(a) and (b) we observe that also the choice of unit or orthonormal initial deviation vectors does not have any practical influence on the evolution of the indices. This becomes more evident from the results of Figure 11(c) where we plot the estimations of the asymptotic GALI values, $\overline{\mathrm{GALI}}_{k}$, obtained from the results of Figures 11(a) and (b) [in the same way that Figure 3(b) was obtained from Figure 3(a)], as the four curves practically overlap.

In Figure 12 we plot the probability density distributions of the coordinates of the unit deviation vectors needed for the evaluation of GALI $_{11}$ for a regular orbit close to SPO1 of Hamiltonian (1) with $N=11$. In particular, we consider the orbit with $H_{N} / N=0.01$, $D=0.12$ in Figure $12(\mathrm{a})$ and $H_{N} / N=0.002$, $D=0.04$ in Figure 12(b). The distributions are created by the coordinates of the set of 11 deviation vectors obtained at 10 snapshots when the $\mathrm{GALI}_{11}$ has reached its asymptotic value. Black curves correspond to initially unit deviation vectors, while gray curves to orthonormal ones. Both curves practically overlap.

The results of Figures 11 and 12 clearly indicate that the initial distribution of the deviation vector coordinates does not play a role in the evolution of GALIs. Thus, we will here continue our investigations by considering only initial unit deviation vectors having their coordinates generated from a uniform distribution.

The distribution of the deviation vectors coordinates evolve in time as we see in Figure 13 where we divide into five time intervals the evolution of $\mathrm{GALI}_{11}$ for a regular orbit close to the stable SPO1 of Hamiltonian (1) for $N=11$, 


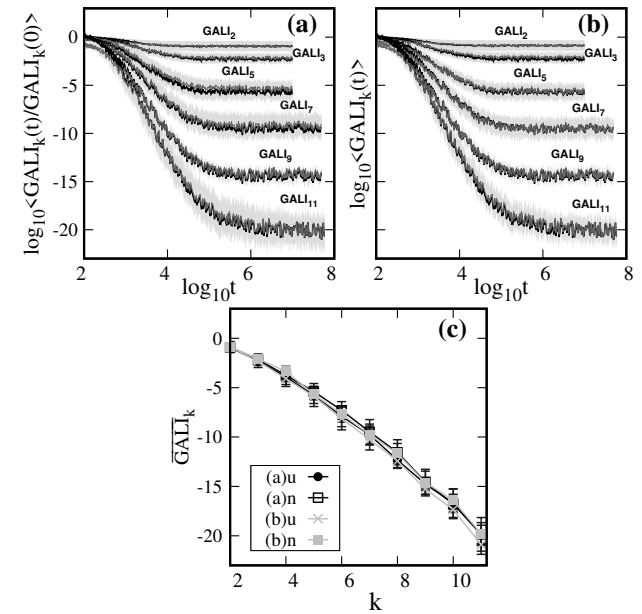

FIG. 11. The time evolution of the average over $n_{v}=10$ sets of initial deviation vectors of (a) $\left\langle\operatorname{GALI}_{k}(t) / \operatorname{GALI}_{k}(0)\right\rangle$ and (b) $\left\langle\operatorname{GALI}_{k}(t)\right\rangle$ for $k=2$, $3,5,7,9,11$, for a regular orbit in the neighborhood of the stable SPO1 $\left(D=0.12, H_{N} / N=0.01\right)$ of Hamiltonian (1) with $N=11$. The coordinates of the initially (a) unit [(b) orthonormalized] deviation vectors were chosen from a uniform (black curves) and a normal distribution (light black curves). The 2 curves practically coincide in panels (a) and (b), while the gray area around them indicate 1 standard deviation. (c) Similar to Figure 3(b) but for the 4 different cases of panels (a) and (b). The cases are denoted in the legend by the panels name, (a) or (b), and by ' $u$ ' or ' $n$ ' for uniform and normal distributions. The 4 curves practically overlap.

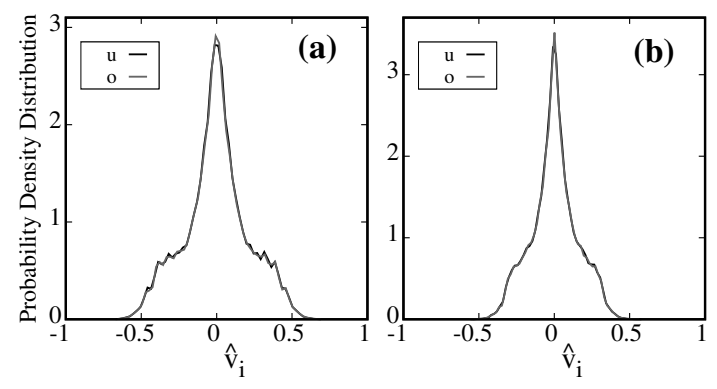

FIG. 12. Probability density distributions of the coordinates $\hat{v}_{i}$ of 11 initially unit (black curves - ' $u$ ') or orthonormalized (gray curves - 'o') deviation vectors for a regular orbit of Hamiltonian (1) with $N=11$ close to the stable SPO1, with (a) $H_{N} / N=0.01$, $D=0.12$, and (b) $H_{N} / N=0.002, D=0.04$. The distributions are created from data obtained from 10 snapshots of the deviation vectors' evolution when the $\mathrm{GALI}_{11}$ values have practically reached their asymptotic behavior. In both panels, the two curves practically overlap. with $H_{N} / N=0.004$ and $D=0.04$ [Figure 13(a)]. Each interval has the same length in logarithmic scale. The corresponding coordinate distributions are shown in Figure 13(b), while in Figure 13(c) we present similar distributions but for another, random set of initial deviation vectors. From Figures 13(b) and (c) we see the evolution of the distributions from a rather spread 'triangular' shape to a more concentrated one, along with clear similarities between the distributions obtained by different initial sets of deviation vectors. Thus, in general, not only the final distribution obtained when GALIs have reached their asymptotic behavior, but also the time evolution of the coordinate distributions is practically independent of the initial choice of deviation vectors.

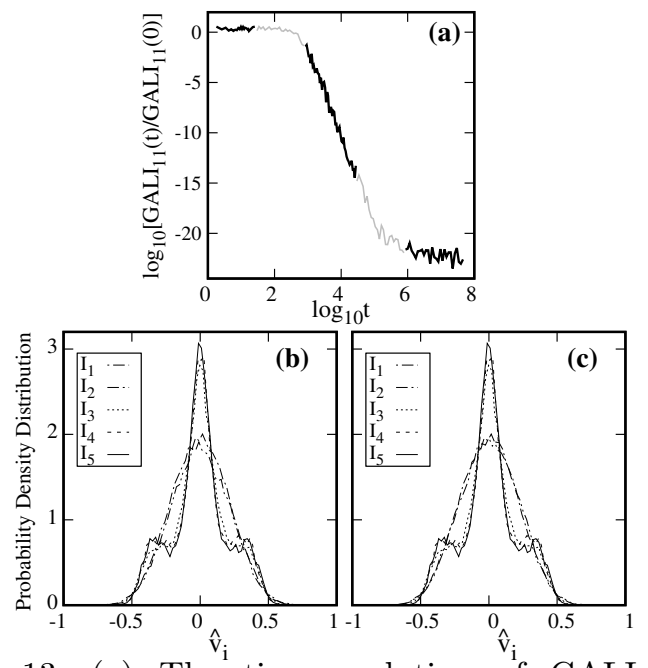

FIG. 13. (a) The time evolution of $\mathrm{GALI}_{11}$ for a regular orbit in the neighborhood of the stable SPO1 orbit of Hamiltonian (1) for $N=11$ with $H_{N} / N=0.004$ and $D=0.04$. The evolution is divided into 5 intervals, $\mathrm{I}_{1}: 0 \leq \log _{10} t<1.5$, $\mathrm{I}_{2}: 1.5 \leq \log _{10} t<3, \quad \mathrm{I}_{3}: 3 \leq \log _{10} t<4.5$, $\mathrm{I}_{4}: 4.5 \leq \log _{10} t<6$ and $\mathrm{I}_{5}: 6 \leq \log _{10} t \leq 7.5$. The coordinate distributions of the 11 unit deviation vectors for the intervals $I_{1}, I_{2}, I_{3}, I_{4}$ and $I_{5}$ are shown in (b) for the deviation vectors used in (a), while in (c) we see results similar to the ones of (b) but for another random set of initial deviation vectors.

In Figures 14 and 15 we investigate the dependence of the final deviation vector coordinate distribution on the regular orbit's distance $D$ from the stable SPO in the case of SPO1 [Figure 14(a)] and SPO2 [Figure 15(a)]. We see that in both cases we have a transition from a rather pointy distribution with high concentrations in the distribution's middle and 


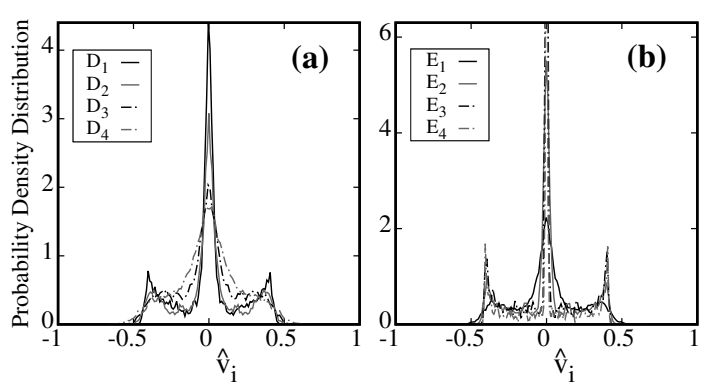

FIG. 14. The final coordinate distributions of the 11 unit deviation vectors used for the computation of $\mathrm{GALI}_{11}$ for regular orbits in the neighborhood of the stable SPO1 of Hamiltonian (1) with $N=11$. The considered regular orbits have (a) $E=H_{N} / N=0.01$ and distances $D(11)$ from the stable SPO1 $D_{1}=0.01$ $D_{2}=0.06, D_{3}=0.12$ and $D_{4}=0.22$, and (b) $E_{1}=0.001$ and $D=0.015, E_{2}=0.01$ and $D=0.015, E_{3}=0.1$ and $D=0.015$, and $E_{4}=0.175$ and $D=0.001$.

edges (small $D$ values) to a more 'triangular' shape as we approach the boundaries of the stability island (large $D$ values). In accordance to this behavior we see in Figures 14(b) and 15(b) that when the orbit's energy density increases towards the SPO's destabilization energy the distributions move to a more pointy and concentrated shape because the regular orbit is located closer to the stable SPO, as we have already pointed out in the discussion of Figure 7.

\section{Summary and discussion}

In this work, we investigated in detail the behavior of the GALI chaos indicator for regular motion of multidimensional Hamiltonian systems. Thus, in this framework, our study builds on and completes previous knowledge/findings on the GALI method $[15,16,18]$ where other aspects of the index were investigated, like for example its behavior for periodic orbits [18]. For this purpose, we considered several regular orbits in the vicinity of two basic SPOs of the FPUT $\beta$ model (1), the so-called SPO1 and SPO2 orbits [36], for various numbers of the system's dof.

We showed that the time evolution of the GALIs, along with the distribution of the coordinates of the deviation vectors needed for their computation, are not influenced by the initial choice of the deviation vectors (Figures 11 and 12). In order to do so, we considered various sets of initial deviation vectors. More specifically, we used unit vectors whose coordinates were
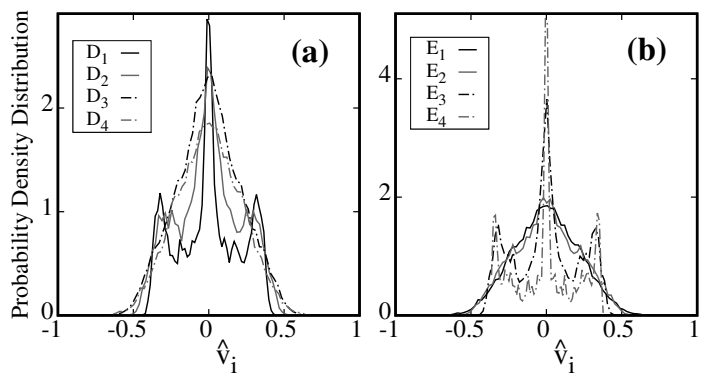

FIG. 15. The final coordinate distributions of the 11 unit deviation vectors used for the computation of $\mathrm{GALI}_{11}$ for regular orbits in the neighborhood of the stable SPO2 of Hamiltonian (1) with $N=11$. The considered regular orbits have (a) $E=H_{N} / N=0.001$ and distances $D(11)$ from the stable SPO2 $D_{1}=0.01$, $D_{2}=0.04, D_{3}=0.08$ and $D_{4}=0.12$, and (b) $E_{1}=0.001$ and $D=0.12, E_{2}=0.004$ and $D=$ $0.12, E_{3}=0.008$ and $D=0.04$, and $E_{4}=0.012$ and $D=0.01$.

drawn randomly from a uniform or a normal distribution. In that case, since the initial value $\operatorname{GALI}_{k}(0)$ is different for each choice of vectors, we registered the evolution of $\left\langle\mathrm{GALI}_{k}(t) / \mathrm{GALI}_{k}(0)\right\rangle$. An alternative option we considered was vectors, whose coordinates were again randomly generated from a uniform or a normal distribution, which were also orthonormalized at the beginning of our numerical simulations, setting in this way $\operatorname{GALI}_{k}(0)=1$. For this choice we followed the evolution of $\left\langle\operatorname{GALI}_{k}(t)\right\rangle$.

The value of $\mathrm{GALI}_{k}$ eventually saturates to a positive value for regular motion on an $N D$ torus for $2 \leq k \leq N$. The asymptotic $\overline{\mathrm{GALI}}_{k}$ values depend on (i) the order $k$ of the index, i.e. GALIs of higher order attain lower values, (ii) the phase space distance $D$ (11) of the regular orbit from the nearby SPO, i.e. the $\overline{\mathrm{GALI}}_{k}$ values increase when $D$ grows, and (iii) the orbit's energy $H_{N}$ (1), or equivalently the energy density $H_{N} / N$, i.e. as we approach the destabilization energy of the SPO the $\overline{\mathrm{GALI}}_{k}$ values decrease. Finally, we also showed that the shape of the coordinate distributions of the deviation vectors depend on $D$ and $H_{N} / N$ (Figures 14 and 15), although these distributions are not influenced by the initial choice of the vectors.

\section{Acknowledgments}

We would like to thank H. Christodoulidi for her fruitful comments and suggestions. H. T. M. was partially funded by the University 
of Cape Town (UCT) International and Refugee Grant, as well as by the Woldia University and MoSHE. Ch. S. acknowledges support by the UCT's Research Committee (URC). We also thank the High Performance Computing facility of UCT (http://hpc.uct.ac.za) and the Center for High Performance Computing (https://www.chpc.ac.za) for providing the computational resources needed for this work.

\section{References}

[1] R.S. MacKay, J.D. Meiss. Hamiltonian Dynamical Systems. (Adam Hilger, Bristol, 1987).

[2] S. Wiggins. Global Bifurcations and Chaos: Analytical Methods. (Springer-Verlag, New York, 1988).

[3] A.J. Lichtenberg, M. A. Lieberman. Regular and Chaotic Dynamics. 2nd edition. (SpringerVerlag, Berlin, 1992).

[4] C. Simó. Hamiltonian Systems with Three or More Degrees of Freedom. Vol. 533, (Nato Science Series C, Springer Netherlands, 1999).

[5] T. Bountis, Ch. Skokos. Complex Hamiltonian Dynamics. (Springer-Verlag, Berlin, 2012).

[6] A.M. Lyapunov. The general problem of the stability of motion. (Taylor and Francis, London, 1992).

[7] V.I. Oseledec. Trans. Moscow Math. Soc. 19, 197 (1968).

[8] G. Benettin, L. Galgani, A. Giorgilli, J.M. Strelcyn. Meccanica. 15, 9 (1980).

[9] G. Benettin, L. Galgani, A. Giorgilli, J.M. Strelcyn. Meccanica. 15, 21 (1980).

[10] Ch. Skokos. Lect. Notes Phys. 790, 63 (2010).

[11] N.P. Maffione, L.A. Darriba, P.M. Cincotta, C.M. Giordano. Cel. Mech. Dyn. Astron. 111, 285 (2011).

[12] L.A. Darriba, N.P. Maffione, P.M. Cincotta, C.M. Giordano. Int. J. Bifurcation Chaos. 22, 1230033 (2012).

[13] D.D. Carpintero, N. Maffione, L. Darriba. Astronomy and Computing. 5, 19 (2014).

[14] Chaos detection and predictability. Eds. Ch. Skokos, G. Gottwald, J. Laskar. Lecture Notes in Physics. Vol. 915. (Springer, Berlin Heidelberg, 2016).

[15] Ch. Skokos, T.C. Bountis, Ch. Antonopoulos. Phys. D. 231, 30 (2007).

[16] Ch. Skokos, T. Bountis, Ch. Antonopoulos. Eur. Phys. J. Spec. Top. 165, 5 (2008).

[17] T. Bountis, T. Manos, H. Christodoulidi. J. Comp. Appl. Math. 227, 17 (2009).
[18] T. Manos, Ch. Skokos, Ch. Antonopoulos. Int. J. Bifurcation Chaos. 22, 1250218 (2012).

[19] Ch. Skokos, T. Manos. Lect. Notes Phys. 915, 129 (2016)

[20] E. Fermi, P. Pasta, S. Ulam, M. Tsingou. Studies of the nonlinear problems. I. Los Alamos Report LA-1940 (1955).

[21] J. Ford Phys. Rep. 213, 271 (1992).

[22] Focus Issue: The 'Fermi-Pasta-Ulam' ProblemThe First Fifty Years. D.K. Campbell, P. Rosenau, G.M. Zaslavsky Eds. Chaos 15, 015101 (2005).

[23] The Fermi-Pasta-Ulam Problem. A Status Report. Ed. G. Gallavotti. (Lecture Notes in Physics. Vol. 728. (Springer, Berlin Heidelberg, 2008).

[24] S. Blanes, F. Casas, A. Farres, J. Laskar, J. Makazaga, and A. Murua, App. Num. Math. 68, 58 (2013).

[25] B. Senyange, Ch. Skokos. Eur. Phys. J. Spec. Top. 227, 625 (2018).

[26] C. Danieli, B. Many Manda, T. Mithun, Ch. Skokos. Math. in Engineering. 1, 447 (2019).

[27] Ch. Skokos, E. Gerlach. Phys. Rev. E. 82, 036704 (2010).

[28] E. Gerlach, Ch. Skokos. Discr. Cont. Dyn. Sys.Supp. 2011, 475 (2011).

[29] E. Gerlach, S. Eggl, Ch. Skokos. Int. J. Bifurcation Chaos. 22, 1250216 (2012).

[30] Ch. Skokos. J. Phys. A. 34, 10029 (2001).

[31] Ch. Skokos, Ch. Antonopoulos, T.C. Bountis, M.N. Vrahatis. Prog. Theor. Phys. Supp. 150, $439(2003)$

[32] Ch. Skokos, Ch. Antonopoulos, T.C. Bountis, M.N. Vrahatis. J. Phys. A. 37, 6269 (2004).

[33] H. Christodoulidi, C. Efthymiopoulos, T. Bountis. Phys. Rev. E. 81, 016210 (2010).

[34] Ch. Skokos. Physica D. 159, 155 (2001).

[35] Ch. Antonopoulos, T. Bountis, Ch. Skokos. Int. J. Bifurcation Chaos. 16, 1777 (2006).

[36] Ch. Antonopoulos, T. Bountis. Phys. Rev. E. 73, 056206 (2006). 